\title{
REFLEXIONES SOBRE LA ORALIZACIÓN DEL SORDO Y ASPECTOS DE SU EDUCACIÓN
}

\author{
REFLECTIONS ON THE ORALIZATION OF THE DEAF AND ASPECTS OF THEIR \\ EDUCATION
}

\author{
Ana Carolina Martins dos Santos \\ Licenciada en Letras \\ Universidad Federal de Río de Janeiro \\ anamartins-96@hotmail.com
}

\begin{abstract}
Resumen: Este artículo busca crear un espacio de reflexión, a partir de lecturas que abordan más íntimamente el tema del individuo sordo y la relación que se establece entre la psicolingüística y la lengua de señas. Tradicionalmente, el trato de la fonoaudiología con los sordos ha estado subvencionada por la perspectiva oralista, apoyada por la normalización de la oralidad y centrada en prácticas alejadas de los usos sociales del lenguaje. Por lo tanto, revisaremos los factores psicosociales y las concepciones históricas que aclaran las actitudes hacia los sordos desde la antigüedad, y examinaremos las razones de los cambios en el enfoque educativo, desde el oralismo a la comunicación total y al bilingüismo. De igual manera, se intenta pensar cómo se dan los aspectos de la educación del niño sordo ya que la sordera es vista como un paradigma pedagógico. Para lograr un mayor nivel de conciencia y llevarla a la práctica es importante considerar las condiciones objetivas y subjetivas, el contexto socio-económicocultural y las formas de pensar de los diversos segmentos de la sociedad.
\end{abstract}

Palabras claves: reflexión; individuo; psicolingüística; sordera; conciencia

Abstract: This article seeks to create a space for reflection, based on readings that address more intimately the issue of the deaf individual and the relationship that is established between psycholinguistics and sign language. Traditionally, the treatment of the deaf by phonoaudiology has been subsidized by the oralist perspective, supported by the normalization of orality and focused on practices far from the social uses of language. Therefore, we will review the psychosocial factors and historical conceptions that elucidate the attitudes towards the deaf since antiquity and examine the reasons for the changes in the educational approach, from oralism to total communication and bilingualism. Likewise, we will try to think about how the aspects of deaf children's education take place since deafness is seen as a pedagogical paradigm. To achieve a higher level of awareness and to put it into practice it is important to consider the objective and subjective conditions, the socio-economic-cultural context and the ways of thinking of the various segments of society.

Keywords: reflection; individual; psycholinguistics; deafness; consciousness 


\section{INTRODUCCIÓN}

Este artículo busca crear un espacio de reflexión, a partir de lecturas que abordan más íntimamente el tema del individuo sordo y la relación que se establece entre la psicolingüística y la lengua de señas, sobre los elementos que abarcan todo el contexto del proceso de oralización del sordo como sus desafíos, sus consecuencias y las críticas, considerando el transcurso histórico de la sordera, de la lengua de señas y del oralismo como instrumento para la inserción del sordo en el contexto social.

Tradicionalmente, el trato de la fonoaudiología con los sordos ha estado subvencionada por la perspectiva oralista, apoyada por la normalización de la oralidad y centrada en prácticas alejadas de los usos sociales del lenguaje. Por lo tanto, revisaremos los factores psicosociales y las concepciones históricas que dilucidan las actitudes hacia los sordos desde la antigüedad, y examinaremos las razones de los cambios en el enfoque educativo, desde el oralismo a la comunicación total y al bilingüismo.

De igual manera se intenta pensar cómo se dan los aspectos de la educación del niño sordo ya que la sordera es vista como un paradigma pedagógico. De hecho, cuando ese paradigma se explicita, aparece junto el aspecto negativo de ser sordo tal que, en esa sociedad, significa un enfrentamiento constante con lo desconocido. Para lograr un mayor nivel de conciencia y llevarla a la práctica es importante considerar las condiciones objetivas y subjetivas, el contexto socioeconómico-cultural y las formas de pensar de los diversos segmentos de la sociedad.

Para dicha reflexión se tuvieron en cuenta consideraciones desde una perspectiva histórica, médica y del propio sujeto en cuestión para que fuera posible construir un análisis crítico.

\section{LAS CONCEPCIONES HISTÓRICAS SOBRE LA SORDERA}

A través de una comprensión histórica de la sordera se puede llegar a lo que hoy conocemos como la lengua de señas. En el siglo IV a.C., Aristóteles suponía que todos los procesos involucrados en el aprendizaje ocurrían a través de la audición y que, en consecuencia, los sordos serían menos educables que los ciegos. En la Edad Media, se suponía que los sordos no tendrían acceso a la salvación, ya que, según Pablo en la Epístola a los Romanos, la fe proviene del oír la palabra de Cristo. A este propósito, sin embargo, hay que reconocer que, siglos más tarde, sería esa misma preocupación para la salvación de los sordos que acabaría motivando a religiosos en todo el mundo a trabajar con sordos buscando rescatar sus señales.

En los siglos XVIII y XIX, los filósofos del lenguaje seguían diseminando la idea de que el sordo no podía aprender y pensar. En su antropología desde una perspectiva pragmática, después de resaltar que el carácter semiótico no icónico de los sonidos del lenguaje es la forma más hábil de denominar las cosas, Kant (1793) concluyó que los sordos "nunca pueden alcanzar más que un análogo de la analogía" (1980, p. 49), y que incluso después de aprender a sentir los movimientos de los órganos del habla, el sordo "nunca llegará a los conceptos generales porque las señales que él necesitaría para ello nunca serían capaces de representar una generalidad" (1980, p. 54). El fundador de la Psicología Experimental, Wilhelm Wundt (1911), fue el único académico en tratar la lengua de señas como un lenguaje en su propio derecho. 
Mientras tanto en la lingüística, la lengua de señas no era considerada como un objeto de estudio. Saussure (1916/1987) enfatizaba la arbitrariedad de las relaciones entre el signo y su referente, y la iconicidad de ciertos signos era vista como prueba de su inferioridad. En la época se concebía la lengua de señas como una forma inferior de comunicación compuesta de un vocabulario limitado de señales equivalentes a la mera gesticulación mímica o a la pantomima, sin estructuras. Una abstracción limitada a una representación holística de ciertos aspectos concretos de la realidad.

\section{PSICOLINGÜÍSTICA Y LA LENGUA DE SEÑAS}

Las concepciones históricas, descritas anteriormente, sobre la sordera nos ayudan a entender las actitudes que se han demostrado hacia los sordos, desde la antigüedad clásica hasta la actualidad. Ya que durante mucho tiempo en la lingüística se pensó que los señas no eran más que mera mímica y pantomima, indignas de un estudio lingüístico.

Hoy en día, un gran número de psicolingüistas se han dedicado a comprender el procesamiento de los lenguajes de signos. La evolución de la psicolingüística ha llevado a la expansión de la investigación para contemplar los procesos heterocíticos del lenguaje verbal. Sin embargo, el hecho de que ahora podamos visualizar las transformaciones lingüísticas que han sufrido los lenguajes de signos, atestigua que el cerebro humano es una máquina incomparable capaz de lidiar con cadenas ininterrumpidas de significado. Además, los factores externos influyen en la configuración interna, de ahí la importancia del entorno social que difunde la información comunicativa y lingüística.

Kruse (1853) afirma que:

La lengua de señas es el verdadero equipamiento de la vida mental del sordo-mudo; él piensa y se comunica sólo por este medio, y él recibe por este mismo medio los conceptos y las ideas [...] Precede cualquier otro lenguaje, y abriendo camino hacia el pensamiento, permite al sordo alcanzar, comprender a la palabra y a la propia idea de lenguaje. La lengua de señas es un medio indispensable de comunicación entre el profesor y el alumno, y es de enorme valor en el aula para la explicación de conceptos y palabras. No sólo abre el camino a la enseñanza inicial, sino que también ofrece un apoyo continuo para el proceso de orientación y explicación. (p. 183-184)

Se puede entender que las lenguas de señas son sistemas abstractos de reglas gramaticales, propias a las comunidades sordas de los países que las utilizan. Al igual que las lenguas habladas, las lenguas de señas no son universales: cada país presenta su propia lengua.

Son lenguas espaciales, es decir, la realización de estas lenguas no se establece a través del canal oral-auditivo, sino a través de la visión y la utilización del espacio, a diferencia de las lenguas orales. La diferencia en la modalidad determina el uso de mecanismos sintácticos específicos diferentes de los utilizados en las lenguas orales.

Entrando en el campo de la adquisición de la lengua de señas por un niño sordo está la cita de Carvalho (2015) que comenta: 
En efecto, la adquisición de la lengua prosigue sin entrenamiento explícito por parte de los usuarios de la lengua ya competentes. Los niños naturalmente alcanzan fluidez comunicativa en cualquier lengua accesible a ellos, siempre y cuando sean expuestos a esa lengua sobre una base regular y frecuente. (p.136)

Fernandes y Correia (2005) lo corroboran al afirmar que:

Propiciar a la persona sorda la exposición a una lengua lo más temprano posible, obedeciendo a las fases naturales de su adquisición es fundamental para su desarrollo, privarla de ese derecho, bajo cualquier argumento, es no respetar a esa persona en su integridad. (p. 18)

A través de tales afirmaciones cabe señalar que las diferencias en la modalidad entre las lenguas orales-auditivas y las lenguas gestuales-visuales no obstruyen el proceso de adquisición de una lengua. Es prácticamente imposible al sordo hablar de forma natural, teniendo en vista el bloqueo sensorial en relación al input lingüístico sonoro que lo rodea. Aunque los sordos pueden ser enseñados a hablar una lengua, nunca serán capaces de comprenderla tan bien como una persona oyente. Por otro lado, los niños sordos, expuestos a la lengua de señas, adquieren de forma natural tal lengua.

Un niño sordo que nace en un ambiente donde los padres utilizan la lengua de señas adquirirá esa lengua de la misma forma en que los niños oyentes adquieren una lengua oral. Así, los niños sordos adquieren la lengua de señas sin ninguna instrucción especial. Ellos comienzan a producir señales, más o menos a la misma edad en que los niños oyentes comienzan a hablar, y atraviesan las mismas etapas de desarrollo en la adquisición de las lenguas naturales. Por lo tanto, si el lenguaje humano es universal en el sentido de que todos los seres humanos poseen la capacidad para adquirir una lengua, no es sorprendente que las lenguas de señas se desarrollen entre personas sordas.

Cuando se trata de la adquisición se ve históricamente cuán difícil era entender todo ese proceso complejo ya que en esa época se pensaba que establecer una comunicación con los sordos era imposible. Tal pensamiento ha cambiado a lo largo del tiempo y mucho se estudió sobre el tema, y lo que se percibe es que la gran mayoría de los niños sordos son hijos de padres oyentes, que muchas veces no saben o saben muy poco sobre el lenguaje de señas y sobre las comunidades de sordos. De este modo, Rodriguero (2013), basado en los estudios de SchmidGiovannini (1980), explica que

El primer paso que debe dar una familia que tiene un hijo sordo es tratar de integrarlo como miembro activo de la comunidad familiar. Esto facilitará que el niño sordo se integre en la comunidad de personas que escuchan. Con el apoyo de la familia, el niño sordo tendrá menos dificultades para vivir con los oyentes, lo que le permitirá ser un miembro activo de esta sociedad. (p. 23) 


\section{ASPECTOS CULTURALES Y SOCIALES}

Todo lo que se ha tratado hasta este momento revela cuán importante es ayudar a los padres, en aceptar la deficiencia de sus niños, ya que eso implica la salud emocional de ambas partes. Este hecho demuestra cómo nuestra sociedad es ajena al tema de la sordera y cuánto se hace necesario integrarla a esa realidad que por muchos es olvidada. Tomando en cuenta la perspectiva del propio sujeto en cuestión (un individuo sordo), este texto reafirma la importancia de insertar, cuanto antes, el niño sordo en un contexto que estimule su desarrollo pleno y natural en la lengua de señas, abordando la oralidad como segunda lengua y mirando a un individuo bilingüe.

A partir de ese punto se puede plantear las cuestiones del ámbito bilingüe para el individuo sordo, reflexionando desde la cita de Carvalho (2005):

El bilingüismo se establece como el uso regular de dos o más lenguas, aunque no haya una competencia necesariamente igual en alguna de ellas. El bilingüismo como el uso cotidiano de al menos dos lenguas por un individuo, puede considerarse un sistema en movimiento en niños y adultos, ya que la utilización y la habilidad de dos lenguas pueden cambiar, dependiendo de las oportunidades de usarlas y exposición a otros usuarios de aquéllas mismas. Aquí, ser bilingüe se justifica socialmente en tanto que aprender otra lengua proporciona al individuo la capacidad de adentrarse en el contexto de otra cultura. (p. 137)

Lo que desarrolla sobre ese asunto es que el individuo sordo bilingüe es fluido en su lengua materna y aprende a través de la enseñanza sistemática una lengua hablada (que aprende a hacer la lectura de los articuladores primarios de esta modalidad) o un sistema de escritura que representa el lenguaje oral. Sin embargo, hay una serie de variables que hacen de una comunidad sorda un grupo no homogéneo en cuanto a la fluidez y dominio de estos sistemas orales. Algunos factores que interfieren en este proceso son: 1) los niveles de sordera; 2) el tiempo de exposición a los sistemas; entre otros.

El individuo, por el hecho de no tener acceso a los sonidos del habla, generalmente comete errores visuales que revelan la mediación de los signos de su lenguaje visual y, por tanto, el mayor desafío para el bilingüismo es tratar con la discontinuidad entre la escritura alfabética y el lenguaje de señas. Como la operación de sistemas de representación externa (es decir, la escritura) siempre se realiza desde el sistema de procesamiento interno, es natural que el niño sordo trate de hacer uso de su señalización interna en asistencia a la lectura y a la escritura. En tanto que el niño oyente utiliza las propiedades fonológicas y fono articulatorias que constituyen la forma de su habla interna, los sordos tienden a recurrir a las propiedades visuales que constituyen la forma de su señalización interna. También están aquellos que, a pesar de la falta de acceso fonológico a la lengua oral, aprenden a utilizar las pistas fono articulatorias, el desarrollo de la lectura de labios y la producción oral del sistema hablado.

El tema del bilingüismo nos lleva más allá, en un plano que de abstracto pasó al concreto y que nos hace llegar a comprender la historia de la oralización. Según Giroto; Cardoso; Azevedo; Kano y Seno (2007) el abordaje oralista en la educación de sordos siempre fue el más utilizado por la visión clínico-terapéutica de la sordera. El discurso médico reconocido es poseedor de 
poder y ha negado enfáticamente la lengua de señas en la vida de los sordos. Lo que acarreó varios daños a la comunidad sorda.

Toda esa temática del proceso de oralización viene desde la segunda mitad del siglo XVIII, en que había dos métodos de enseñanza de sordos: el método en París, que se basaba en un sistema artificial de signos; y el método alemán, que enfatizaba el desarrollo de la oralización. A partir del Congreso de Milán en 1880, el método oralista se volvió dominante. En consecuencia, la educación del sordo se redujo a la enseñanza de la oralización, los profesores sordos fueron expulsados, la lengua de señas fue prohibida y la comunidad sorda fue excluida de la política de las instituciones de enseñanza, por ser considerada un peligro para el desarrollo del lenguaje oral. Aparentemente como resultado de la concentración exclusiva de la educación en la oralización, el nivel educativo del sordo cayó muy por debajo del de los oyentes. Esto sólo empezó a cambiar en 1980 en todo el mundo, incluso en la propia tierra del método oralista, de donde partió buena parte de las resistencias al avance de la lengua de señas en la educación.

\section{PERSPECTIVA MÉDICO-FONOAUDIOLÓGICA DE LA SORDERA}

Frente a esa causa se levanta la perspectiva médica sobre el tema. Eso nos lleva a pensar sobre ¿Qué hacen los fonoaudiólogos con los niños sordos?, ¿Cuáles son los hechos que presentan frente a la oralización?, ¿Cómo actúan ante la sordera? Entre otras tantas preguntas que surgen. Giroto et al. (2007) exponen que:

La actuación fonoaudiológica enfatiza acciones que dan oportunidades a los intercambios entre los discursos, sea por medio de la lengua de señas, sea por la modalidad oral y/o la ampliación de las habilidades lingüísticas. Tales acciones también se centran en el trabajo con la función auditiva; a la utilización de la lectura labial, en situaciones cerradas y abiertas, de acuerdo con las exigencias de los contextos de uso para tal recurso; a la realización de una evaluación audiológica sistemática; a la adecuación de condiciones para el uso apropiado del aparato de amplificación sonora individual y la participación de los familiares en las actividades desarrolladas. La utilización del lenguaje oral es estimulada, sin embargo, no es priorizada, cabiendo al sordo la opción por la modalidad de lenguaje considerada, por él, más significativa, dependiendo de los contextos cotidianos que se presentan. (p. 4)

Es interesante ver cómo la presencia del fonoaudiólogo juega con lo psicológico del niño, cómo lo estimula a buscar su propio camino, cómo lo hace individuo pensando que también hará parte de un medio colectivo.

En lo que fue relatado en ese texto, los fonoaudiólogos revelan que, actualmente, el trabajo con la práctica escrita del niño sordo no se está realizando en función de las fallas (morfosintácticas) generalmente observadas en sus textos, sino en función de la necesidad que identifican de construir con los niños un sistema de referencia. Como una memoria de lo que se dice en la vida cotidiana. Este es el abordaje fundamental para que el niño tenga sentido de lo que lee y escribe, sentido que se construye en y por la interacción de discursos.

Tales expertos afirman que por el bilingüismo se reconoce que el sordo vive en una situación bilingüe, rodeado por la modalidad oral y por la lengua de señas. De este modo, el individuo sordo puede elegir la lengua que va a utilizar en cada situación lingüística en que se 
encuentre, tomando en cuenta sus interlocutores y las condiciones de uso de estas modalidades lingüísticas de acuerdo con los contextos en que esté. Se percibe que el lenguaje escrito difícilmente es el motivo por lo cual los niños sordos van a la clínica fonoaudiológica, pero la sordera, las alteraciones en los procesos de interlocución del niño sordo con el oyente y las experiencias escolares sin éxito dejan marcas significativas, que interfieren en el proceso de reconocimiento de la lengua escrita y en las prácticas de lectura y escritura. El problema auditivo, la preocupación por la prótesis auditiva, por el habla en el sentido de la articulación o de la consulta del lenguaje oral son los motivos más comunes por los que los padres de un niño sordo buscan al fonoaudiólogo.

Para aprehender la materialidad significante del lenguaje escrito se usa la visión, y ésta es considerada un instrumento y un material imprescindible en función del proceso terapéutico con los niños, principalmente porque el material escrito trae dentro de la sesión posibilidades de diálogo, discusión, conocimiento e información, posibilitando la relación con el lenguaje en los aspectos de los sentidos y significados.

El acceso a la lectura es considerado un factor esencial para permitir al sordo en su lenguaje, hablar de su lugar de sujeto y superar lo que constituye el drama principal del sordo profundo, que es la incapacidad de llegar a la polisemia de la lengua. Por eso, es tan importante la figura de un profesional calificado, el fonoaudiólogo. Según Trenche y Balieiro (2004):

El trabajo con la escritura ya empieza con esa laguna porque (el niño sordo) no tiene todavía un lenguaje real, incluso para los niños que tienen un mejor dominio del lenguaje de signos, ya que la estructura es completamente diferente del portugués, su adquisición es comparable a la adquisición de una segunda lengua y para algunos fonoaudiólogos como lengua extranjera. Sin poder utilizar plenamente la capacidad de pensar verbalmente que proporciona el uso del lenguaje, el proceso de adquisición de la escritura es a menudo experimentado por el niño como "un trabajo largo y duro". (p. 178-179)

A través de eso se explica que el enfoque del trabajo del fonoaudiólogo no incide sobre las técnicas de lectura relacionadas con las actividades de enseñanza de la lengua, las prácticas de ejercicio del uso de las señales gráficas, puntuación y acentuación. Al entender eso se rompen muchos paradigmas formados delante de tal enseñanza, además que se reitera que la misma se centra en cómo posibilitar condiciones para que el niño pueda, poco a poco, vivir la escritura como una experiencia que amplíe la reflexión sobre sí y sobre el mundo, en llevarlo a usar la experiencia que desarrolló en contacto con diversos portadores de texto para que produzca el suyo mismo, en una interacción del discurso, yendo, de esta forma, mucho más allá de las preocupaciones exclusivas con la grafía y la ortografía.

Siguiendo el razonamiento de ese mismo texto, se percibe que los fonoaudiólogos que se dedican a gran parte del desarrollo del individuo con tal deficiencia afirman pautados en vivencias, estudios y casos clínicos que la mayoría de los sordos no se benefician de la oralización y que muchas veces su aspecto lingüístico y educativo es afectado cuando no se prioriza la lengua natural.

Así como hay posiciones en contra hay posiciones a favor de la oralización y eso está muy aclarado en el blog Surdos Usuários da Língua Portuguesa (2015) donde se menciona que: 
Sólo no vemos el motivo para aprender una lengua que no nos será de utilidad y con la que no nos identificamos. Es casi lo mismo que ser obligados a aprender latín, una lengua muerta. Y como el resto de la sociedad no conoce la lengua de señas, como tampoco sabe hablar latín, lo máximo que estamos logrando con esa medida legal "humanitaria" es crear otra diferencia entre nosotros y los oyentes - en donde, ciertamente, nacerá un perjuicio más. (párr. 4)

Estas líneas dejan ver que hay una parte de sordos oralizados que afirma que sólo por la oralización ellos tienen posibilidades e iniciativas ampliadas como cualquier otro ser humano y, por eso, creen que únicamente por esa vía son capaces de ser incluidos en la sociedad, sin que sean discriminados. Por este motivo, no están de acuerdo con el hecho de que la lengua de señas sea la lengua exclusiva y única del sordo. Como parte de la comunidad sorda, ellos afirman que el gran problema de la sordera no es la falta de comunicación, sino la falta de información por parte de la sociedad. Aseguran también que la lengua de señas caracteriza un avance en relación a la problemática del sordo, pero que no resuelve los problemas más graves de información y de comunicación.

Ese Manifiesto es un documento que representa a un grupo de sordos que pasaron por el proceso de oralización y que defienden que esta es la mejor salida para que un deficiente auditivo sea incluido totalmente en la sociedad. Alegan que la lengua de señas, tenida como prioridad en sus vidas, acaba por separarlos de la parte oyente y dejarlos en una posición inferior y minoritaria y que, por consiguiente, no logran una real inserción en los diversos ámbitos sociales. De tal manera se rige la lucha de este grupo que consienta en el aprendizaje de la lengua de señas, pero no quiere que la misma reciba una posición de lengua oficial de los sordos una vez que, para ellos, la oralización también está en cuestión.

Parar para percibir el otro lado de la historia y la otra visión fue importante para la construcción del raciocinio hasta aquí, ya que son ellos quienes viven esa realidad. Sin embargo, se nota que hay un enfoque muy grande en la relación del individuo sordo y su aceptación en el mundo, pero el Manifiesto acaba por dejar de lado la importancia del desarrollo psíquico de ese mismo individuo al aprender la lengua de señas y los beneficios involucrados en la apropiación de un lenguaje propio para él. El argumento expuesto por el grupo de sordos oralizados debe considerarse válido, pero no absoluto; ya que en contraposición se presentan hechos bajo la visión de fonoaudiólogos que mucho esclarecen acerca de la complejidad del asunto.

A partir de ahí se establece un confronte entre opiniones, es difícil equilibrarlas cuando las dos deben ser tratadas como legítimas, una vez que surgen de un mismo punto de partida pero que convergen en diferentes lugares. Se refleja entonces el lado opuesto cuando Giroto et al. $(2007$, p. 1) hacen frente y aseguran que "En consecuencia, gran parte de los sordos no es beneficiada por el oralismo, ya que no se apropian efectivamente de la modalidad oral del lenguaje, ni tampoco de la escritura, cuya enseñanza se basa en estrategias orales".

\section{ACTUALIDAD Y POSIBILIDADES EDUCATIVAS (ESCUELA, FAMILIA, ESTUDIANTE)}

Luego de todo esto, podemos enfocarnos en el factor enseñanza que asume una integración escolar de los sordos, dado que ha sido defendida por poderes oficiales, con un 
discurso que apela a las emociones. Por ende, han tratado de difundir la idea de que es un acto de discriminación colocar a los sordos, así como a cualquier otro tipo de "discapacitado", en escuelas especiales. Este acto se atribuye como un ataque a la modernidad, $\mathrm{o}$ al avance tecnológico, incluso si se desea mantener grupos "aislados". Se defiende la idea de que colocar a los "discapacitados" junto a las personas "normales" es un signo de gran progreso impulsado por la solidaridad. El enfoque está en las concesiones y ajustes que las escuelas e instituciones deben hacer para "recibirlas". La idea es mantener a "todos" juntos para asimilar la diversidad. Sin embargo, lo que no es muy explícito es que la separación del otro puede lograrse, a pesar del enfoque físico, restringiendo la comunicación.

La inclusión de los sordos en las aulas regulares hace imposible que los sordos construyan conocimiento, identidad y cultura a partir de las dos lenguas (la lengua de signos y la lengua oficial del país) y hace imposible que los estudiantes sordos consoliden sus habilidades lingüísticas. No se trata sólo de aceptar el lenguaje de signos, sino de hacerlo posible, porque todo trabajo pedagógico que considere el desarrollo cognitivo tiene que considerar la adquisición de una primera lengua natural (este es el eje fundamental del "bilingüismo", como lo defendemos). De lo contrario, ¿cómo establecerá el niño contacto con el mundo de las representaciones que lo rodean? ¿Cómo tejerá sus propios significados? Por el contrario, si el niño sordo tiene un lenguaje natural, tendrá la base para la adquisición de un segundo lenguaje, porque tendrá las condiciones óptimas para el desarrollo de su cognición, su autoestima y su identidad.

Ahora, a los defensores de la "integración escolar ecuánime", podríamos preguntar: ¿es posible tener una escuela donde haya el mismo número de niños sordos y oyentes? ¿Es posible tener el mismo número de profesores y oyentes sordos, y que todos hablen con fluidez ambos idiomas? Ahora, incluso si esto fuera posible, todavía no habría madres, padres, abuelos y hermanos sordos para distribuir a todos los niños sordos... De todos modos, una integración escolar ecuánime se ve bien sólo en los discursos.

Cuando se defiende el lenguaje de signos como primera lengua, no se está diciendo que el desarrollo cognitivo dependa exclusivamente del dominio de un idioma, sino que se está creyendo que el dominio de un idioma garantiza mejores recursos para las cadenas neuronales implicadas en el desarrollo de los procesos cognitivos. Así, objetivamente, lo que pretenden los defensores del "bilingüismo" es garantizar el dominio de un idioma para dar bases sólidas al desarrollo cognitivo del individuo (Fernandes y Correia, 2005, p. 49). Sin embargo, también requiere el cuidado de no pretender una exclusividad para la lengua de señas (como si los sordos no pudieran aprender la lengua de la comunidad mayoritaria), es decir: "Defendemos un bilingüismo, no un monolingüismo al revés" (Fernandes y Correia 2005, p. 50).

Se desprende de ahí todo lo investigado, como la articulación del sordo que tiende a ser recibida con extrañamiento por los oyentes, pues muchos de los que han logrado aprender a oralizar se sienten inhibidos y desanimados en hacerlo fuera de su círculo de amistad en el día a día. A pesar de todos sus esfuerzos, en el método oralista las habilidades de lectura y escritura de los sordos tienden a limitarse al nivel de la tercera serie del primer grado. $\mathrm{Y}$, como consecuencia de las limitaciones en el desarrollo de competencias lingüísticas de lectura y escritura, tienden a tener déficit también en otras áreas de conocimiento y materias escolares.

Por otra parte, no tiene sentido la búsqueda constante por la reafirmación de una identidad sorda, incluso las definidas por las teorías actuales vigentes en la Educación de Sordos. Teóricamente, hay una amplia gama de identidades, pero eso sigue en las peleas ideológicas 
entre sordos y educadores de la sordera que defienden la lengua de señas como primera y la otra parte se compone de sordos oralizados de destacadas proyecciones y estilos de vida intelectual en función del éxito del método oralista.

De modo que lo entendido aquí destituye todo lo que fue argumentado por los sordos oralizados, en función de comprobaciones biológicas que se dan en el desarrollo de un niño sordo.

Pero como cada individuo es único en sí mismo, no se puede decir que los oralistas están ciertos, tampoco exaltar la eficiencia del método cultural de los sordos no oralizados. El objetivo debe ser el de concebir una plataforma formativa que sea capaz de suplir todas las necesidades del individuo sordo que, debido a su diferencia, lo ponen en una situación de inferioridad ante la sociedad, mayoritaria, oyente.

No hay una concordancia en cuál es la mejor técnica de formación de la mentalidad del sordo o de un individuo capaz de sobrevivir a las adversidades de la vida sin la preocupación imperiosa que envuelve la idea de su deficiencia. Si, por un lado, no es posible educar efectivamente al sordo por el sistema de la oralización, sobre la pena de obstruir o incluso retrasar su crecimiento cultural inicial, entre otras consecuencias, tampoco podemos tomar parte de la lengua de señas por poner la capacidad lingüística del sordo en desacuerdo con la cultura dominante. Siendo que esta cultura dominante urge ser incorporada bajo pena de no disponer de un crecimiento profesional mayor, el sordo que no domina esta cultura permanece en segundo plano, en servicios secundarios y de bajos ingresos, por lo que es necesario un nuevo concepto que atienda la necesidad primordial de hacer la vida del sordo más productiva tanto como extender su rayo de alcance en la sociedad.

Toda esa vía trazada hasta aquí busca un complemento que sea como cierre para que el fin se dé, de hecho, en los aspectos de la educación del niño sordo, así como explicita Giroto et al. (2007, p. 5) "El aprendizaje de la modalidad oral es deseado, pero no puede ser perseguido como el único objetivo educativo y comunicativo, ni como la única posibilidad de minimizar las diferencias causadas por la sordera." De la misma manera se lo puede comparar a través de lo que corrobora Lier-de Vitto (1994) ya que tales aspectos educativos deben partir del ámbito familiar, en dónde la familia es orientada a no hablar por sus hijos, tampoco intentar interpretar o adivinar lo que dicen, en lugar de eso se recomienda que ellos hablen con sus hijos como lo harían a cualquier niño oyente. Así que la práctica del proceso educativo tanto en la escuela como en casa ha sido, en general, desarrollada a partir de la idea de un individuo hipotético. Se tiende a hacer generalizaciones.

En detrimento de una visión más realista pues independiente de ser sorda, cada persona presenta características de personalidad, producto de su trayectoria histórica-económica-social. Muchas veces, no se considera la diversidad característica que hay en un grupo. El grupo de sordos, como el grupo de los oyentes, presenta características internas de etnia, de clase, de género, de religión, entre otras cosas que pueden provocar inseguridad, incluso conflictos y divergencias, llegando a influir en la personalidad del individuo.

Al buscar un punto en que se pueda dialogar con el tema de la identidad del niño sordo Pereira (2011) afirma:

Me llama la atención el hecho de que niños con la misma deficiencia auditiva y pertenecientes a familia de nivel socio-económico-cultural semejante no parecen beneficiarse del mismo modo de una misma orientación. Tales diferencias [...] parecen 
depender más de la forma como la familia representa el niño sordo como un individuo capaz de desarrollar un lenguaje. (p. 67)

Las identidades, ante todo, no deben privilegiar una condición de ser sordo, ya que es un proceso de auto aceptación como persona. Y esto no depende necesariamente de una conciencia sorda para entenderla. La identidad es histórica y camina a lo largo de una vida llena de marcas por lo que los sordos se convierten en seres sociales y participativos de la sociedad/ un ser social y participativo de la sociedad. Debe haber un ideal pedagógico que analice antes quién es esa persona sorda, cuáles son sus diferencias individuales, sobre qué bases ideológicas están sedimentadas sus concepciones de mundo. Y para que ese ideal pedagógico sea adecuadamente suplementado, es preciso respetar las características individuales de cada grupo de sordos, según los cuales responden a determinados patrones de condicionamiento, dependiendo de las diferentes asociaciones teóricas que les pueden atribuir.

Los sordos oralizados y no oralizados generalmente presentan diferentes raíces de concepción del mundo. Mientras estos últimos están más cerca de una "masificación" de la cultura sorda, que tiene en la lengua de señas su manifestación mayor de cultura; los oralizados se acercan más a las manifestaciones de la cultura oyente, donde se privilegia la habilidad del habla y la eficacia en lectura labial. Además de eso, Pereira (2011) afirma que:

[...] aunque no escuchen, niños sordos sufren el efecto de la lengua hablada por la madre. La lengua, que se procesa a través de la modalidad oral, tiene efecto de significación del niño para sí mismo, de los objetos y de los demás. Tal efecto se manifiesta a través de la incorporación tanto en el proceso gestual como del habla. (p. 73)

Por eso, las características pedagógico-educativas exigidas por ambos grupos son diferenciadas y el nivel de dificultades de comunicación dentro de las aulas son, en teoría, mucho mayor que la presentada cuando hay sordos estudiando en presencia de intérpretes de lengua de señas, de ahí la principal dificultad de los sordos es el acompañamiento de las clases. En cualquier caso, siempre habrá la barrera de la comunicación en ambos grupos, en mayor o menor grado, además de las curiosidades ajenas y las miradas reprobadoras de algunos, por puro prejuicio velado.

\section{CONCLUSIONES}

Entendemos que los sordos forman una minoría cultural no mejor ni peor que otras minorías, sólo diferente. Las minorías culturales suelen estar oprimidas y dominadas por las culturas que ejercen el mayor poder, sin embargo, es importante destacar que, una minoría cultural no siempre es una minoría cuantitativa, porque hay minorías cuantitativas que ejercen un mayor poder sobre los significados y los sentidos, ya sea el poder económico, el poder de decisión, la enunciación, etc.

Por lo tanto, para experimentar todo esto en una realidad concreta, es necesario entender que la propuesta educativa bilingüe es la que más posibilita el desarrollo integral del niño sordo, ya que respeta su condición proporcionando que ésta se desarrolle primero en su lengua natural. 
El contacto con la comunidad sorda proporciona la formación de la identidad del sordo y confiere a éste la capacidad de dominio del lenguaje, pudiendo así dar significado al mundo. La intención no es aislar al individuo sordo a un grupo, sino permitir que primero éste tenga la oportunidad de adquirir el lenguaje, así como el oyente.

Finalmente, todo ese proceso tiene que permitir y hacer real la inserción social del sordo, que camine efectivamente por la interacción social establecida básicamente por las posibilidades comunicativas. La inclusión social sólo ocurre verdaderamente cuando el sujeto pudo haberse constituido como tal, a partir de sus relaciones familiares, que van siendo gradualmente ampliadas en los ambientes escolar y social.

Las elecciones de los caminos a recorrer con los sordos se centran en la familia, con el apoyo, conocimiento y orientación de profesionales especializados. Pero, por encima de cualquier determinación externa, él debe ser autónomo para optar por su integración a la sociedad oyente a través del desarrollo de la comunicación oral, o a la comunidad de sordos, a través de la adquisición del lenguaje de señas naturales, o su inserción en las dos culturas.

\section{BIBLIOGRAFÍA}

Carvalho, J. C. B. (2015). Considerações sobre a aquisição da Libras e do Português escrito por surdo em um contexto bilíngue. Revista Cadernos de Estudos e Pesquisas na Educação Básica, 1(1), 135-148.

Fernandes, E., y Correia, C. (2005). Bilinguismo e Surdez: A evolução dos conceitos no domínio da linguagem. En F. Eulália (Ed.), Surdez e Bilinguismo. (pp.103) Porto Alegre, Brasil: Editorial Mediação.

Giroto, C., Cardoso, L., Azevedo, A., Kano, C., y Seno, M. (Octubre de 2007). Práticas Fonoaudiológicas com o surdo sob a perspectiva bilíngue. Núcleo de Estudos da FFCUNESP (Presidencia). Enfoque interdisciplinar de Atendimento Bilíngue a Surdos, Familiares e Professores. Simposio llevado a cabo en el IV Congresso Brasileiro Multidisciplinar de Educação Especial, Londrina, Brasil.

Kant, I. (4 Ed.). (1980). Anthropologie in pragmatischer Hinsicht. Hamburg, Alemania Editorial: Leipzig: I. Müller.

Kruse, O. (1853). Über Taubstumme und Taubstummenanstalten nebst Notizen aus meinem Reisetagebuch. Selbstverlag.

Lier-de Vitto, M. F. (2 Ed.). (1994). Fonoaudiologia: no sentido da Linguagem. São Paulo, Brasil: Editorial Cortez.

Pereira, M. C. da C. (2011). Conversação entre mães ouvintes e crianças surdas. Cadernos De Estudos Lingüísticos, 33, 67-73. doi: https://doi.org/10.20396/cel.v33i0.8637097 
Rodriguero, C. R. B. (1 Ed.). (2013). A Família e o filho surdo: uma investigação acerca do desenvolvimento psicológico da criança segundo a abordagem histórico-cultural. Curitiba, Brasil: Editorial CRV.

Surdos Usuários da Língua Portuguesa. (21 de noviembre de 2015). O Manifesto dos Surdos Oralizados. Sulp - surdos usuários da língua portuguesa: manifesto dos surdos oralizadosdocumento histórico. Recuperado de www.sulpsurdosusuariosdalinguaportuguesa.blogspot.com

Trenche, M. C. B., y Balieiro, C. R. (2004). A escrita e prática fonoaudiológica com crianças surdas/deficientes auditivas. Distúrbios da Comunicação, 16(2), 175-80. 